\title{
THE TRACE OF THE HEAT KERNEL IN LIPSCHITZ DOMAINS
}

\author{
RUSSELL M. BROWN
}

\begin{abstract}
We establish the existence of an asymptotic expansion as $t \rightarrow 0^{+}$ for the trace of the heat kernel for the Neumann Laplacian in a bounded Lipschitz domain. The proof of an asymptotic expansion for the heat kernel for the Dirichlet Laplacian is also sketched. The treatment of the Dirichlet Laplacian extends work of Brossard and Carmona who obtained the same result in $C^{1}$-domains.
\end{abstract}

A classical question in analysis asks: What is the relationship between the spectrum of the Laplacian on a domain and the geometry of the domain? This question has been studied by many authors including $[1,3,7,11,12,14,15$, $17,19]$. One approach to this problem proceeds by considering the trace of the heat semigroup on $D$,

$$
\operatorname{tr}(g)(t)=\int_{D} g(t, X, X) d X=\sum_{j} e^{-\lambda_{j} t}
$$

where $g$ is the heat kernel on $D$ and $0 \leq \lambda_{1} \leq \lambda_{2} \leq \lambda_{3} \cdots$ is the sequence of eigenvalues for $-\Delta$ on $D$.

One attempts to give an asymptotic expansion for $\operatorname{tr}(g)$ near $t=0$ whose coefficients are quantities associated with the geometry of $D$. Thus one can deduce geometrical information about $D$ from knowledge of the spectrum.

In this paper, we will address this question under minimal smoothness assumptions on the boundary of $D$. In particular, we will obtain asymptotic expansions for $\operatorname{tr}(g)$ when the order of smoothness of the boundary corresponds exactly to the order of accuracy of the asymptotic expansion.

In order to state our main theorem, we need one definition: By a Lipschitz domain, we mean a bounded connected open set whose boundary may be locally described as the graph of a Lipschitz function. Our main result is

Main Theorem. Suppose that $D \subset \mathbf{R}^{n}$ is a Lipschitz domain, then the heat kernel for the Laplacian with Dirichlet boundary conditions on $\partial D$ satisfies

$$
\operatorname{tr}(g)(t)=(4 \pi t)^{-n / 2}\left(|D|-\frac{\sqrt{\pi t}}{2} \mathscr{H}^{n-1}(\partial D)+o\left(t^{1 / 2}\right)\right) .
$$

Received by the editors August 14, 1991.

1991 Mathematics Subject Classification. Primary 35P20.

The author was partially supported by the NSF and by the Commonwealth of Kentucky through the Kentucky EPSCoR program. 
If $g$ is the heat kernel with Neumann boundary conditions, then we have the expansion

$$
\operatorname{tr}(g)(t)=(4 \pi t)^{-n / 2}\left(|D|+\frac{\sqrt{\pi t}}{2} \mathscr{H}^{n-1}(\partial D)+o\left(t^{1 / 2}\right)\right) .
$$

We note several related results. When the domain is $C^{1}$, the portion of our theorem concerning Dirichlet boundary conditions has been proved by $\mathrm{J}$. Brossard and R. Carmona [5]. When the domain is smooth or is a polygon, then results of this type are well known, see $[1,4,12,15]$. These authors either use explicit calculations to study polygons or results on boundary value problems which fail in nonsmooth domains.

We also mention the work of van den Berg [3] who proves the expansion

$$
\left|\operatorname{tr}(g)(t)-(4 \pi t)^{-n / 2}\left(|D|-\frac{\sqrt{\pi t}}{2} \mathscr{H}^{n-1}(\partial D)\right)\right| \leq \frac{C_{n} t^{1-(n / 2)}|D|}{R^{2}}
$$

for the Dirichlet heat kernel in a domain which satisfies a uniform interior and exterior sphere condition of radius $R$. This is of interest because the error term is of the same order as the degree of smoothness that is assumed on the boundary of $D$.

We mention the recent work of Lapidus and Fleckinger-Pelle $[13,14]$. They consider Dirichlet and Neumann Laplacians on domains which have so-called fractal boundaries. More precisely, they assume that the Minkowski dimension of $\partial D$ is greater than $n-1$ and obtain asymptotic formulae for the distribution of the eigenvalues. From their results, one can deduce that

$$
(4 \pi t)^{n / 2} \operatorname{tr}(g)(t)=|D|+O\left(t^{(n-d) / 2}\right)
$$

as $t \rightarrow 0^{+}$. Here $d$ is the Minkowski dimension of $\partial D$. If we use the dimension of the boundary as a measure of smoothness, then their error term corresponds exactly to the order of smoothness assumed on $\partial D$.

Finally, we observe that there is a good deal of information available for more exotic domains (see $[2,18])$. For special classes of domains, one can obtain more informatiom about the heat kernel. However, within the scale of $C^{k, \alpha}$-domains, our results are sharp. For example, one can construct a Lipschitz domain, $D$, such that for all $\varepsilon>0$, we have

$$
\varlimsup_{t \rightarrow 0^{+}} t^{-1 / 2-\varepsilon}\left|(4 \pi t)^{n / 2} \int_{D} g(t, X, X) d X-\right| D\left|+\frac{\sqrt{\pi t}}{2} \mathscr{H}^{n-1}(\partial D)\right|>0
$$

where $g$ denotes the Dirichlet heat kernel in $D$.

Our argument breaks naturally into several steps. First, we need some simple ideas from geometric measure theory to construct and estimate the size of a good set of points. Near this good set of points, we are able to give good estimates for the heat kernel of $D$. The first part of the argument is identical for the Dirichlet and Neumann heat kernels. In the second part of the argument, we consider the Dirichlet and Neumann cases separately. We will concentrate on the case of Neumann boundary conditions since this part of the argument, for Dirichlet boundary conditions, is similar to the work of J. Brossard and R. Carmona's treatment of $C^{1}$-domains [5]. The ideas needed to estimate the heat kernel with 
Neumann boundary conditions come from [8] where B. Dahlberg and C. Kenig study the Neumann problem for Laplace's equation in Lipschitz domains.

\section{SOME PRELIMINARY RESULTS}

We begin by defining the notion of the Minkowski content of a set. We let $\omega_{s}=\pi^{s / 2} / \Gamma(s / 2+1)$ and for $0 \leq d \leq n$, we define

$$
\mathscr{M}^{d}(E)=\lim _{r \rightarrow 0^{+}} \frac{\mid\{X \in D: \operatorname{dist}(X, E)<r\}}{\frac{1}{2} \omega_{n-d} r^{n-d}} .
$$

We remark that this might be better called the Minkowski content of $E$ with respect to $D$, but in the interest of brevity, we will refer simply to the Minkowski content. We note that if $E \subset \partial D$, the boundary of a Lipschitz domain, and $E$ is closed, then $\mathscr{M}^{n-1}(E)=\mathscr{H}^{n-1}(E)$ [10, Theorem 3.2.39] where $\mathscr{H}^{d}$ denotes the more familiar Hausdorff measure. In our next proposition and throughout this paper, we will use $\delta(X)$ to denote the distance between $X$ and $\partial D$.

Proposition 1.1. Let $D$ be a measurable subset of $\mathbf{R}^{n}$ whose boundary is of finite d-dimensional Minkowski content. Suppose that $f:(0, \infty) \rightarrow \mathbf{R}$ is locally $C^{1}$, is bounded, satisfies $\lim _{t \rightarrow \infty} f(t)=0$ and

$$
\int_{0}^{\infty}\left|f^{\prime}(t)\right| t^{n-d} d t<\infty
$$

Under these assumptions, we have

$$
\lim _{\varepsilon \rightarrow 0^{+}} \frac{1}{\varepsilon^{n-d}} \int_{D} f(\delta(X) / \varepsilon) d X=\mathscr{M}^{d}(\partial D) \frac{\omega_{n-d}}{2}(n-d) \int_{0}^{\infty} f(s) s^{n-d-1} d s .
$$

Proof. Let $\psi_{\varepsilon}(r)=\varepsilon^{d-n}|\{X \in D: \delta(X)<\varepsilon r\}|$. It is fairly easy to see that

$$
\varepsilon^{d-n} \int_{D} f(\delta(X) / \varepsilon) d X=\int_{0}^{\infty} f(r) d \psi_{\varepsilon}(r)
$$

We integrate by parts in the Riemann-Stieltjes integral on the right and obtain

$$
\int_{\eta}^{R} f(r) d \psi_{\varepsilon}(r)=-\int_{\eta}^{R} f^{\prime}(r) \psi_{\varepsilon}(r) d r+\psi_{\varepsilon}(R) f(R)-\psi_{\varepsilon}(\eta) f(\eta) .
$$

The boundary term at infinity vanishes because $\lim _{t \rightarrow \infty} f(t)=0$ and $\psi_{\varepsilon}(R)$ is bounded in $R$, for each fixed $\varepsilon$. The boundary term at zero vanishes because $\psi_{\varepsilon}(r) \rightarrow 0$ as $r \rightarrow 0^{+}$and $f$ is bounded. Next, observe that $\psi_{\varepsilon}(r)$ converges to $\frac{1}{2} \omega_{n-d} r^{n-d} \mathscr{M}^{d}(\partial D)$ pointwise as $\varepsilon \rightarrow 0^{+}$and that $\psi_{\varepsilon}(r) \leq C r^{n-d}$ uniformly for all $\varepsilon>0$. This and the Lebesgue dominated convergence theorem imply that

$$
\lim _{\varepsilon \rightarrow 0^{+}} \int_{0}^{\infty} \psi_{\varepsilon}(r) f^{\prime}(r) d r=\frac{1}{2} \omega_{n-d} \mathscr{M}^{d}(\partial D) \int_{0}^{\infty} f^{\prime}(r) r^{n-d} d r .
$$

To complete the proof of the proposition, we integrate by parts again. To see that the boundary term at infinity vanishes, we need the estimate

$$
R^{n-d}|f(R)| \leq \int_{R}^{\infty}\left|f^{\prime}(s)\right| s^{n-d} d s
$$

which follows by writing $f$ as the integral of its derivative. The boundary term at zero vanishes because $f$ is bounded. 
Definition. We say that $G \subset \partial D$ is $(\varepsilon, r)$-good if for each $P \in \partial D$, the unit inner normal $\nu(P)$ exists and

$$
B(P, r) \cap \partial D \subset\{X:|(X-P) \cdot \nu(P)|<\varepsilon|X-P|\} .
$$

Given an $(\varepsilon, r)$-good set $G$, we construct the sawtooth region

$$
\mathscr{G}=\bigcup_{P \in G} \Gamma_{r}(P, 2 \varepsilon)
$$

where $\Gamma_{r}(P, \varepsilon)$ is the truncated cone

$$
\{X:(X-P) \cdot \nu(P)>(1-\varepsilon)|X-P|\} \cap B(P, r) .
$$

Our next proposition is rather technical. It will be used to estimate the size of the bad set where we do not have good information about the heat kernel $g(t, X, X)$.

Proposition 1.3. Let $\varepsilon<\frac{1}{2}$ and suppose that $G$ is a measurable, $(\varepsilon, r)$-good subset of $\partial D$, the boundary of a Lipschitz domain. Then there exists $s_{0}$ depending on $\partial D$ and $G$ so that for $s<s_{0}$, we have

$$
|\{X: \delta(X)<s\} \backslash \mathscr{G}| \leq s\left[\mathscr{H}^{n-1}(\partial D \backslash G)+\varepsilon\left(3+\mathscr{H}^{n-1}(\partial D)\right)\right] \text {. }
$$

Proof. We let $\mathscr{G}_{s}=\{X: \delta(X)<s\} \cap \mathscr{G}$ and we will show that there exists $s_{0}$ so that

$$
\left|\mathscr{G}_{s}\right| \geq s\left(\mathscr{\ell}^{n-1}(G)-\varepsilon\left(2+\mathscr{L}^{n-1}(G)\right), \quad s<s_{0} .\right.
$$

This and our observation that $\mathscr{H}^{n-1}(\partial D)$ and $\mathscr{M}^{n-1}(\partial D)$ agree are sufficient to imply the conclusion after possibly choosing $s_{0}$ smaller.

To establish (1.4), we choose $\nu_{1}, \ldots, \nu_{N} \in \mathbf{S}^{n-1}$ and $F_{1}, \ldots, F_{N} \subset G$, closed sets, which satisfy $F_{i} \cap F_{j}=\varnothing$ for $i \neq j, \mathscr{C}^{n-1}\left(G \backslash \bigcup_{i=1}^{N} F_{i}\right)<\varepsilon$, and $\left|\nu(P)-\nu_{i}\right|<\varepsilon$ when $P \in F_{i}$. We have $P+\rho \nu_{i} \in \mathscr{G}_{\rho}$ when $\rho<r$ and $P \in F_{i}$. First, we claim that the map $P \rightarrow P+\rho \nu_{i}$ is injective when $P \in F_{i}$ and $0<\rho<r$. To see this, suppose that $P-Q=(\rho-\sigma) \nu_{i}$ for $P, Q \in F_{i}$ and $\rho, \sigma \in(0, r)$. We have

$$
\begin{aligned}
|\rho-\sigma| & =\left|(\rho-\sigma)\left(\nu_{i} \cdot \nu_{i}\right)\right| \\
& =\left|(P-Q) \cdot \nu_{i}\right| \leq|(P-Q) \cdot \nu(P)|+\left|(P-Q) \cdot\left(\nu_{i}-\nu(P)\right)\right| \\
& \leq \varepsilon|P-Q|+\varepsilon|P-Q|=2 \varepsilon|\rho-\sigma| .
\end{aligned}
$$

The second inequality is our assumption that $P$ is $(\varepsilon, r)$-good and our choice of $\left|\nu_{i}-\nu(P)\right|$. This gives a contradiction if $\varepsilon<\frac{1}{2}$.

To estimate the size of $\mathscr{G}_{s}$, we let $\gamma$ denote the minimum of $2 \operatorname{dist}\left(F_{i}, F_{j}\right)$ for $i \neq j$ and let $s_{0}=\min (\gamma, r)$. When $s<s_{0}$, we have

$$
\begin{aligned}
\left|\mathscr{G}_{s}\right| & \geq \sum_{i=1}^{N}\left|\left\{P+t \nu_{i}: P \in F_{i}, 0<t<s\right\}\right| \\
& \geq s(1-\varepsilon) \sum_{i=1}^{N} \mathscr{H}^{n-1}\left(F_{i}\right) \geq s\left[\mathscr{P}^{n-1}(G)-\varepsilon\left(2+\mathscr{H}^{n-1}(G)\right)\right] .
\end{aligned}
$$

The second inequality may be deduced from the area formula [10, Theorem 3.2.3] applied to the map $(P, \rho) \rightarrow P+\rho \nu_{i}$. We have proven (1.4). 


\section{OUTLINE OF THE PROOF}

We let $g_{+}(t, X, X)$ denote the Neumann heat kernel and $g_{-}(t, X, X)$ denote the Dirichlet heat kernel. In this section, we reduce the proof of our theorem to several estimates for these kernels. One feature of Lipschitz domains is that we are unable to prove sufficiently good estimates for $g_{ \pm}$which are uniform near all of $\partial D$. Instead, we divide $\partial D$ into a good and a bad set. Near the good set, we will be able to give precise estimates for $g_{ \pm}$. We will use Proposition 1.3 to show that the bad set is small and makes a negligible contribution to the trace of $g_{ \pm}$. We summarize our estimates in

Proposition 2.1. Let $D$ be a Lipschitz domain and let $\varepsilon>0$. There exists $r>0$ and an $(\varepsilon, r)$-good set $G$ such that if $\mathscr{G}$ is as in (1.2), then we have

$$
\begin{array}{r}
\left|(4 \pi t)^{n / 2} g_{ \pm}(t, X, X)-1 \mp \exp \left(\frac{-\delta(X)^{2}}{t}\right)\right| \leq C \varepsilon, \\
\text { for } X \in \mathscr{G} \cap\{\delta(X)<\sqrt{t / \varepsilon}\} .
\end{array}
$$

$$
\begin{gathered}
0 \leq g_{-}(t, X, X) \leq(4 \pi t)^{-n / 2}, \quad X \in D . \\
0 \leq g_{+}(t, X, X) \leq C\left(1+t^{-n / 2}\right), \\
\left|1-(4 \pi t)^{n / 2} g_{-}(t, X, X)\right| \leq C \exp \left(\frac{-\delta(X)^{2}}{C t}\right), \\
\left|1-(4 \pi t)^{n / 2} g_{+}(t, X, X)\right| \leq C_{\eta}\left(\frac{\sqrt{t}}{\delta(X)}\right)^{\eta} \exp \left(\frac{-\delta(X)^{2}}{C t}\right) .
\end{gathered}
$$

In $(2.4+), \eta$ is any positive number.

We note that the estimate $(2.3-)$ follows from the maximum principle and $(2.3+)$ is established by E. B. Davies in [9, Theorem 3.2.9]. Estimate (2.2-) is similar to [5, Lemma 3.8]. The estimate (2.4-) is well known and follows from the maximum principle (see [5, Remark 3.2]). Thus we still need to establish $(2.2+)$ and $(2.4+)$. We will do this in $\S \S 3$ and 4 . For the moment we accept the estimates of Proposition 2.1 and show how they may be used to prove our main theorem.

Proof of the Main Theorem. We choose $\varepsilon>0$ and will show that for $t$ sufficiently small, we have

$$
\left|(4 \pi t)^{n / 2} \int_{D} g_{ \pm}(t, X, X) d X-\right| D\left|\mp \frac{\sqrt{\pi t}}{2} \mathscr{H}^{n-1}(\partial D)\right| \leq C \sqrt{\varepsilon t}
$$

where $C$ depends only on $D$.

Proposition 2.1 gives us a good set $G$ and we let $\mathscr{G}$ be the sawtooth region constructed in (1.2). We write $D=\bigcup_{i=1}^{3} \mathscr{O}_{i}(t)$ where the sets $\mathscr{O}_{i}$ are defined by

$$
\begin{aligned}
& \mathscr{O}_{1}(t)=\{X: \delta(X)<\sqrt{t / \varepsilon}\} \cap \mathscr{G}, \\
& \mathscr{O}_{2}(t)=\{X: \delta(X)<\sqrt{t / \varepsilon}\} \backslash \mathscr{G}, \\
& \mathscr{O}_{3}(t)=\{X: \delta(X) \geq \sqrt{t / \varepsilon}\} .
\end{aligned}
$$


We begin by using Proposition 1.1 to obtain that

$$
\begin{aligned}
\int_{\mathscr{O}_{3}(t)} \exp \left(\frac{-\delta(X)^{2}}{t}\right) d X & \leq \exp \left(\frac{-1}{2 \varepsilon}\right) \int_{D} \exp \left(\frac{-\delta(X)^{2}}{2 t}\right) d X \\
& \leq C \sqrt{t} \exp \left(-\frac{1}{2 \varepsilon}\right) .
\end{aligned}
$$

From Proposition 1.3, we have for $t$ small that

$$
\int_{\mathscr{O}_{2}(t)} \exp \left(\frac{-\delta(X)^{2}}{t}\right) d X \leq C \sqrt{\varepsilon t}
$$

Thus from $(2.2+)$ and Proposition 1.1, we obtain that

$$
\left|\int_{\mathcal{O}_{1}(t)} 1-(4 \pi t)^{n / 2} g_{ \pm}(t, X, X) d X \mp \frac{\sqrt{\pi t}}{2} \mathscr{H}^{n-1}(\partial D)\right| \leq C \sqrt{\varepsilon t}
$$

for $t$ sufficiently small. On $\mathscr{O}_{2}(t)$, we have

$$
\left|\int_{\mathscr{O}_{2}(t)} 1-(4 \pi t)^{n / 2} g_{ \pm}(t, X, X) d X\right| \leq C\left|\mathscr{O}_{2}(t)\right| \leq C \sqrt{\varepsilon t},
$$

by $(2.3 \pm)$ and Proposition 1.3.

Finally, on $\mathscr{O}_{3}(t)$, we may use $(2.4+)$ and Proposition 1.1 to obtain

$$
\left|\int_{\mathcal{O}_{3}(t)} 1-(4 \pi t)^{n / 2} g_{+}(t, X, X) d X\right| \leq \frac{C_{\eta}}{\varepsilon^{\eta / 2}} \exp \left(-\frac{1}{C \varepsilon}\right)
$$

for $\eta>0$.

The estimate (2.4-) gives the slightly better estimate

$$
\left|\int_{\mathscr{O}_{3}(t)} 1-(4 \pi t)^{n / 2} g_{-}(t, X, X) d X\right| \leq C \exp \left(\frac{-1}{C \varepsilon}\right) \sqrt{t} .
$$

Our asymptotic expansion for $\operatorname{tr}\left(g_{+}\right)$now follows from (2.7), (2.8), and (2.9) and the expansion for $\operatorname{tr}\left(g_{-}\right)$follows from (2.7), (2.8), and (2.10).

\section{ESTIMATES FOR THE INITIAL-NEUMANN PROBLEM}

In this section, we will establish estimates for the initial-Neumann problem in a Lipschitz cylinder problem with inhomogeneous lateral data. We will show that on the lateral boundary, the map from Neumann data to solution maps $L^{p}((0,1) \times \partial D)$ into $L^{p *}((0,1) \times \partial D)$ where $p^{*}=p(n+1) /(n+1-p)$, $1<p<n+1$. Of course these exponents are dictated by homogeneity: The $L^{p}$-properties of this map are identical to that of a parabolic fractional integral of order 1 . We also note that for $p \in(n+1, n+1+\varepsilon)$, we map into Hölder spaces. This will be important since these pointwise estimates will allow us to study $g_{+}(t, X, Y)$ at $Y=X$ and $X$ near the boundary.

We begin by recalling a special case of Davies's result on the Neumann heat kernel [9, Theorem 3.2.9]. We drop the + since we will only consider the Neumann heat kernel. 
Theorem 3.1. Let $D$ be a Lipschitz domain, then the Neumann heat kernel, $g_{+}$ for $D$ satisfies

$$
0 \leq g(t, X, Y) \leq C\left(1+t^{-n / 2}\right) \exp \left(\frac{-|X-Y|^{2}}{C t}\right) .
$$

We will also need

Corollary 3.2. Let $g$ be the Neumann heat kernel for a Lipschitz domain $D$. Let $r=|t|^{1 / 2}+\left|X-Q_{1}\right|$ and suppose that $|t-s|^{1 / 2}+\left|Q_{1}-Q_{2}\right|<r / 2$, then we have

$$
\begin{aligned}
& \left|g\left(t, X, Q_{1}\right)-g\left(s, X, Q_{2}\right)\right| \\
& \quad \leq C\left(\frac{|t-s|^{1 / 2}+\left|Q_{1}-Q_{2}\right|}{r}\right)\left(1+t^{-n / 2}\right) \exp \left(\frac{-\left|X-Q_{1}\right|^{2}}{2 C t}\right) .
\end{aligned}
$$

Proof. Fix a point $P \in \partial D$, a coordinate system on $\mathbf{R}^{n}$ and a Lipschitz function $\phi: \mathbf{R}^{n-1} \rightarrow \mathbf{R}$ so that $\partial D \cap B(P, r)=\left\{\left(X^{\prime}, X_{n}\right): X_{n}>\phi\left(X^{\prime}\right)\right\} \cap B(P, r)$. Near $P$, we may extend $g(t, X, \cdot)$ by setting

$$
\tilde{g}(s, X, Y)=\left\{\begin{aligned}
& g(s, X, Y), \quad Y \in D \\
& g(s, X,\left(Y^{\prime}, 2 \phi\left(Y^{\prime}\right)-Y_{n}\right), \\
&\left(Y^{\prime}, 2 \phi\left(Y^{\prime}\right)-Y_{n}\right) \in B(P, r) \cap D .
\end{aligned}\right.
$$

The function $\tilde{g}(\cdot, X, \cdot)$ is a solution of a divergence form parabolic equation, hence the Nash-Moser [16] result on Hölder continuity applies to prove the corollary.

For more details on the reflection used to extend $g$ see [6] or the earlier work of Dahlberg and Kenig [8].

We take the opportunity to introduce some additional notation that we will need throughout the rest of this paper. We let $\Delta(P, r)=B(P, r) \cap \partial D$ denote a surface ball in $\partial D$. We let $I(P, r)=\left(0, r^{2}\right) \times \Delta(P, r)$ denote a parabolic ball contained in $(0, \infty) \times \partial D$.

The following lemma is taken from the proof of Lemma 3.1 in [6]. This depends on ideas from [8]. We repeat a simplified version of the proof for the convenience of the reader.

Lemma 3.3. Let $a$ be supported in $I(P, r)$, satisfy $\iint a=0$ and $\|a\|_{L^{2}(I(P, r))} \leq$ $\mathrm{Cr}^{-(n+1) / 2}$. If $u$ is the solution of the initial-Neumann problem with data $a$, then we have

$$
|u(t, X)| \leq C\left(\frac{r}{\sqrt{t}}\right)^{\alpha}\left(1+t^{-n / 2}\right), \quad t \geq 2 r^{2} .
$$

Proof. We may write

$$
\begin{aligned}
u(t, X) & =\int_{0}^{r^{2}} \int_{\partial D} g(t-s, X, Q) a(s, Q) d Q d s \\
& =\int_{0}^{r^{2}} \int_{\partial D}[g(t-s, X, Q)-g(t, X, P)] a(s, Q) d Q d s
\end{aligned}
$$

since $a$ has mean 0 . By Corollary 3.2, we may estimate the absolute value of the expression in square brackets by $\left(1+t^{-n / 2}\right)(r / \sqrt{t})^{\alpha}$ on the support of $a$. 
Lemma 3.4. Let $a$ be supported in $I\left(Q_{0}, r\right)$ and suppose that

$$
a \in L^{p}((0, \infty) \times \partial D), \quad p>n+1 .
$$

Let $u$ denote the solution of the Neumann problem with data $a$. Then

$$
|u(t, X)| \leq c\|a\|_{L^{p}((0, \infty) \times \partial D)} r^{1-(n+1) / p} .
$$

Proof. From the estimate of Theorem 3.1, it follows that

$$
\left(\int_{I\left(Q_{0}\right)} g(t-s, X, Q)^{p^{\prime}} d Q d s\right)^{1 / p^{\prime}} \leq C r^{1-(n+1) / p}
$$

when $p>n+1$ and $p^{\prime}=p /(p-1)$. This lemma follows from this, Hölder's inequality and the representation for $u$ in the previous lemma.

Remark. We note that if we use the estimates for $g_{+}$from Corollary 3.2, we may obtain that: $\partial u / \partial \nu \in L^{p}((0,1) \times \partial D)$ implies that $u \in C^{1-(n+1) / p}((0,1) \times$ $\partial D)$ for $p \in(n+1, n+1+\varepsilon)$ where $\varepsilon$ depends on the $\alpha$ in Corollary 3.2.

Our last lemma provides a proof of the estimate $(2.4+)$ in Proposition 2.1.

Lemma 3.5. Let $g_{+}(t, X, Y)$ be the Neumann heat kernel for a Lipschitz domain $D$. Then for each $\varepsilon>0$, we have

$$
\left|g(t, X, X)-(4 \pi t)^{-n / 2}\right| \leq C_{\varepsilon} t^{-n / 2-\varepsilon} \delta(X)^{2 \varepsilon} \exp \left(\frac{-\delta(X)^{2}}{C t}\right) .
$$

Proof. We let

$$
v(t, X, Y)=g(t, X, Y)-p(t, X, Y) .
$$

The function $w(t, Y) \equiv v(t, X, Y)$ solves the initial-Neumann problem

$$
\left\{\begin{array}{l}
\partial_{t} w-\Delta w=0 \\
w(0, Y)=0 \\
\frac{\partial w}{\partial \nu}(t, Q)=\frac{\partial}{\partial \nu(Q)} p(s, X, Q) .
\end{array}\right.
$$

We have the representation formula

$$
w(t, Y)=\int_{0}^{t} \int_{\partial D} g_{+}(t-s, Y, Q) \frac{\partial}{\partial \nu(Q)} p(s, X, Q) d Q d s .
$$

Since we have an explicit formula for $p$, it is easy to see that

$$
\left|\frac{\partial}{\partial \nu} p(s, X, Q)\right| \leq C s^{-n / 2-1 / 2} \exp \left(\frac{|X-Q|^{2}}{8 s}\right) .
$$

If we use this and the estimate for $g$ in Theorem 3.1, then we easily obtain the desired bound for $w$.

\section{Completion of the proof of Proposition 2.1}

In this section, we will construct the good set $G$ and establish the estimate $(2.2+)$ for the Neumann heat kernel. After doing this, we will make a few more remarks about the proof of (2.2-) for Dirichlet boundary conditions. The remaining statements in Proposition 2.1 have already been established. 
Throughout this section, we assume that $\varepsilon>0$ is fixed. We let $G(r)$ denote the set of points $P \in \partial D$ such that the inner normal $\nu(P)$ exists and the conditions (4.1) and (4.2) below hold.

$$
\begin{gathered}
|(P-Q) \cdot \nu(P)|<\varepsilon|P-Q|, \quad Q \in \Delta(P, r), \\
\int_{\Delta(P, s)}|\nu(P)-\nu(Q)|^{n+2} d Q \leq \varepsilon^{n+2} \mathscr{C}^{n-1}(\Delta(P, s)), \quad s<r .
\end{gathered}
$$

By Rademacher's theorem on the almost everywhere differentiability of Lip schitz functions and the Hardy-Littlewood maximal theorem, almost every point of $\partial D$ lies in some $G(r)$ for $r$ small. Hence, we may find $r_{0}$ so that $G \equiv G\left(r_{0}\right)$ satisfies

$$
\mathscr{C}^{n-1}\left(\partial D \backslash G\left(r_{0}\right)\right)<\varepsilon .
$$

Given $\varepsilon$, the set $G\left(r_{0}\right)$ described above and the associated sawtooth region (see (1.2)) will be the good set where the estimate $(2.2+)$ will hold.

Given $X \in \mathscr{G}$, we choose $\widehat{X} \in \partial D$ so that $X \in \Gamma_{r}(\hat{X}, 2 \varepsilon)$. Then we may define $X^{*}$ by the relation

$$
X^{*}-\widehat{X}=\widehat{X}-X .
$$

Of course $\widehat{X}$, and hence $X^{*}$, is not unique but this will not hinder us in proving the estimate $(2.2+)$.

As before, we let

$$
v(t, X, Y)=g(t, X, Y)-p(t, X, Y) .
$$

We claim that $v(t, X, X)$ is close to $p\left(t, X, X^{*}\right)$. To be precise, we will show that for $X \in \mathscr{G}$ and $t$ small, we have

$$
\left|p\left(t, X, X^{*}\right)-v(t, X, X)\right| \leq C \varepsilon t^{-n / 2} .
$$

Since ||$X-X^{*}|-2 \delta(X)| \leq C \varepsilon \delta(X)$ the estimate (2.2+) follows from (4.4).

Thus, we turn to the proof of (4.4). On the boundary, we have that

$$
\frac{\partial v}{\partial \nu}(\cdot, \cdot, X)=-\frac{\partial p}{\partial \nu}(\cdot, \cdot, X),
$$

thus our first step is to collect estimates for the Neumann boundary data of $v$. We let

$$
f(s, Q)=\frac{\partial}{\partial \nu(Q)} p(s, Q, X)+\frac{\partial}{\partial \nu(Q)} p\left(s, Q, X^{*}\right)
$$

denote the error between the Neumann boundary data of $v$ and of $p\left(\cdot, \cdot, X^{*}\right)$. For $2 \delta(X)<\rho<r$, we claim that

$$
\int_{I(\widehat{X}, \rho)} f(s, Q) d Q d s \leq C \varepsilon
$$

where $\hat{X}$ is as in (4.3) and $C$ is a dimensional constant. We can estimate the size of $f$ by

$$
\int_{I(\widehat{X}, 2 \rho) \backslash I(\widehat{X}, \rho)}|f(s, Q)|^{p} d Q d s \leq C \varepsilon^{p} \rho^{(n+1)(1-p)}
$$


where $p=n+2$ and

$$
\int_{I(\widehat{X}, 2 \delta(X))}|f(x, Q)|^{p} d Q d s \leq C \varepsilon^{p} \delta(X)^{(n+1)(1-p)} .
$$

To prove (4.5), we introduce domains $D_{\rho}^{+}=D \cap B(\widehat{X}, \rho)$ and $D_{\rho}^{-}=\bar{D}^{c} \cap$ $B(\widehat{X}, \rho)$. We apply Green's formula in the domains $D_{\rho}^{ \pm}$and obtain that

$$
\begin{aligned}
\int_{I_{\rho}} f(s, Q) d Q d s= & \int_{0}^{\rho^{2}} \int_{\Sigma_{\rho}^{-}} \frac{\partial}{\partial \nu(Q)} p\left(s, Q, X^{*}\right) d Q d s \\
& -\int_{0}^{\rho^{2}} \int_{\Sigma_{\rho}^{+}} \frac{\partial}{\partial \nu(Q)} p(x, Q, X) d Q d s \\
& +\int_{D_{\rho}^{+}} p\left(\rho^{2}, Y, X^{*}\right) d Y-\int_{D_{\rho}^{-}} p\left(\rho^{2}, Y, X\right) d Y
\end{aligned}
$$

where $\Sigma_{\rho}^{+}=\partial D_{\rho}^{+} \cap D$ and $\Sigma_{\rho}^{-}=\partial D_{\rho}^{-} \cap \bar{D}^{c}$. We let $B_{\rho}^{ \pm}=B(\widehat{X}, \rho) \cap\{Y:(Y-$ $\left.\widehat{X}) \cdot\left(X-X^{*}\right) \gtrless 0\right\}$. By symmetry we have

$$
\int_{B_{\rho}^{+}} p(s, Y, X) d Y=\int_{B_{\rho}^{-}} p\left(s, Y, X^{*}\right) d Y .
$$

Hence we can bound the difference of the last two integrals in (4.8) by

$$
\left[\sup _{Y \in B(\widehat{X}, \rho)} p(s, Y, X)+p\left(s, Y, X^{*}\right)\right]\left[\left|D_{\rho}^{+} \Delta B_{\rho}^{+}\right|+\left|D_{\rho}^{-} \Delta B_{\rho}^{-}\right|\right] \leq C \varepsilon .
$$

In this last estimate, $\Delta$ denotes symmetric difference of two sets. Note that we have used that $\widehat{X}$ is $(\varepsilon, r)$-good to estimate the measure of the sets appearing in this expression. Similar considerations produce the same estimates for the first two integrals appearing in (4.8).

Next, we prove (4.6). We begin by observing that our assumption that $\widehat{X}$ is $(\varepsilon, r)$-good implies that

$$
|\nu(\widehat{X}) \cdot(\widehat{X}-Q)| \leq \varepsilon|Q-\widehat{X}|, \quad Q \in \Delta(\widehat{X}, r),
$$

and that

$$
\left\|X-Q|-| X^{*}-Q\right\| \leq \varepsilon|\widehat{X}-Q|, \quad Q \in \Delta(\widehat{X}, r) .
$$

Finally, since $X-\widehat{X}=\widehat{X}-X^{*}$, we may write

$$
\begin{aligned}
&\left.\iint_{I_{2 \rho} \backslash I_{\rho}}\left|\nu(Q) \cdot \nabla_{Q} p(s, Q, X)+\nabla_{Q} p\right|\left(s, Q, X^{*}\right)\right|^{p} d Q d s \\
& \leq C_{p} \iint_{I_{2 \rho} \backslash I_{\rho}}\left|(\nu(Q)-\nu(\widehat{X})) \cdot\left(\nabla p(x, Q, X)+\nabla p\left(x, Q, X^{*}\right)\right)\right|^{p} \\
&+\mid \frac{1}{2(4 \pi)^{n / 2} s^{(n / 2)+1}}(\nu(\widehat{X}) \cdot(X-Q)) \\
&\left.\cdot\left(\exp \left(\frac{-|X-Q|^{2}}{4 s}\right)-\exp \left(\frac{-\left|X^{*}-Q\right|^{2}}{4 s}\right)\right)\right|^{p} \\
&+\left|\frac{1}{(4 \pi)^{n / 2} s^{(n / 2)+1}}(\nu(\widehat{X}) \cdot(\widehat{X}-Q)) \exp \left(\frac{-\left|X^{*}-Q\right|^{2}}{4 s}\right)\right|^{p} d Q d s
\end{aligned}
$$


where we are using $I_{\rho}$ to denote $I(\widehat{X}, \rho)$. The first term is small by virtue of the estimate (4.2) for $\nu-\nu(\widehat{X})$. The second is small because of (4.10) and the third is small because of (4.9). The estimate (4.7) is proven in a similar manner.

We are now ready to give the proof of the estimate (4.4) which describes the asymptotic behavior of $v(t, X, X)$. We first consider $X$ such that $2 \delta(X)<$ $\sqrt{t}$. We let $\rho_{k}=2^{k} \delta(X)$ and $I_{k}=I\left(\widehat{X}, \rho_{k}\right)$ for $k \geq 1$. We choose $N$ so that $2^{N} \delta(X) \leq \sqrt{t}<2^{N+1} \delta(X)$ and let

$$
m_{k}(s, Q)=\chi_{I_{k}} f_{I_{k}} f(s, Q) d Q d s, \quad k \geq 1,
$$

and define $m_{0}=0$. We define $R_{k}=I_{k} \backslash I_{k-1}$ for $k \geq 2$ and put $R_{1}=I_{1}$. Now, we may decompose $f$ into "atoms" by letting

$$
a_{k}=\chi_{R_{k}} f+m_{k-1}-m_{k}, \quad k=1, \ldots, N,
$$

and setting $a_{N+1}=m_{N}+\chi_{I_{N}^{c}} f$. It is clear that $f=\sum_{k=1}^{N+1} a_{k}$. One may check that

$$
\int a_{k}=0, \quad k=1, \ldots, N
$$

and

$$
\int\left|a_{k}\right|^{p} \leq C \varepsilon \rho_{k}^{(n+1)(1-p)}, \quad k=1, \ldots, N,
$$

where again $p=n+2$. If we let $w_{k}$ be the solution of the initial-Neumann problem with data $a_{k}$, then from Lemma 3.3, we have

$$
\left|w_{k}(t, X)\right| \leq C \varepsilon 2^{\alpha(k-N)} t^{-n / 2}, \quad k=1, \ldots, N .
$$

We may sum this estimate and obtain

$$
\sum_{k=1}^{N}\left|w_{k}(t, X)\right| \leq C \varepsilon t^{-n / 2} \text {. }
$$

To estimate the contribution from $a_{N+1}$, we again divide $a_{N+1}$ into atoms supported in annular rings by setting $\rho_{k}=2^{k} \sqrt{t}, k \geq 1, R_{k}=I_{k} \backslash I_{k-1}, k \geq 2$ and $R_{1}=I_{1}$. We let

$$
b_{k}=\chi_{R_{k}} a_{N+1}, \quad k=1, \ldots, M,
$$

where $M$ is chosen so that $2^{M} \sqrt{t} \leq r<2^{M+1} \sqrt{t}$. Finally we let $b_{M+1}=$ $\chi_{I_{M}^{c}} a_{N+1}$. If $u_{k}$ is the solution of the initial-Neumann problem with lateral data $b_{k}$, then we obtain that

$$
\left|u_{k}(t, X)\right| \leq C \varepsilon 2^{-n k} t^{-n / 2}, \quad k=1, \ldots, M .
$$

This follows from Lemma 3.4 and (4.6). Finally, we estimate $u_{M+1}$. We observe that

$$
\int_{0}^{t} \int_{\partial D}\left|b_{M+1}(s, Q)\right|^{2} d Q d s \leq \frac{C t \mathscr{H}^{n-1}(\partial D)}{r^{2 n+2}} \exp \left(\frac{-r^{2}}{4 t}\right)
$$

when $\sqrt{t}<r$. From this and the estimate of Lemma 3.4, we obtain

$$
\left|u_{M+1}(t, X)\right| \leq C_{D, r} t \exp \left(\frac{-r^{2}}{4 t}\right) \text {. }
$$


It remains to choose $t$ small to make the quantity on the right bounded by $\varepsilon t^{-n / 2}$. This completes the proof of $(4.4)$ when $\sqrt{t}>2 \delta(X)$. The case $\sqrt{t} \leq$ $2 \delta(X)$ uses the techniques which estimated $w_{N+1}$.

To prove Proposition 2.1 under Dirichlet boundary conditions, only the condition (4.1) is needed to construct the good set $G$. The estimate $(2.2-)$ follows from the ideas used by Brossard and Carmona [5, Lemma 3.8].

\section{REFERENCES}

1. P. B. Bailey and F. H. Brownell, Removal of the log factor in the asymptotic estimates of polyhedral membrane eigenvalues, J. Math. Anal. Appl. 4 (1962), 212-239.

2. M. van den Berg, On the spectrum of the Dirichlet Laplacian for horn-shaped regions in $\mathbf{R}^{n}$ with infinite volume, J. Funct. Anal. 58 (1984), 150-156.

3. $\ldots$, On the asymptotics of the heat equation and bounds on traces associated with the Dirichlet Laplacian, J. Funct. Anal. 71 (1987), 279-293.

4. M. van den Berg and S. Srisatkunarajah, Heat equation for a region in $\mathbf{R}^{2}$ with polygonal boundary, J. London Math. Soc. 37 (1988), 119-127.

5. Jean Brossard and René Carmona, Can one hear the dimension of a fractal?, Comm. Math. Phys. 104 (1986), 103-122.

6. Russell M. Brown, The initial-Neumann problem for the heat equation in Lipschitz cylinders, Trans. Amer. Math. Soc. 320 (1990), 1-52.

7. R. Courant, Über die eigenwerte bei der Differentialgleichungen der Mathematischen Physik, Math. Z. 7 (1920), 1-57.

8. B. E. J. Dahlberg and C. E. Kenig, Hardy spaces and the Neumann problem in $L^{p}$ for Laplace's equation in Lipschitz domains, Ann. of Math. (2) 125 (1987), 437-466.

9. E. B. Davies, Heat kernels and spectral theory, Cambridge Univ. Press, 1989.

10. H. Federer, Geometric measure theory, Springer-Verlag, 1969.

11. V. Ja. Ivrii, Second term of the spectral asymptotic expansion of the Laplace-Beltrami operator on manifolds with boundary, Funct. Anal. Appl. 14 (1980), 98-106.

12. M. Kac, Can one hear the shape of a drum? Amer. Math. Monthly 73 (1966), 1-23, Part II of H. E. Slaught Memorial Papers.

13. M. Lapidus, Fractal drum, inverse spectral problems for elliptic operators and a partial resolution of the Weyl-Berry conjecture, Trans. Amer. Math. Soc. 325 (1991), 465-529.

14. M. L. Lapidus and J. Fleckinger-Pelle, Tambour fractale: vers une resolution de la conjecture de Weyl-Berry pour les valeurs propres du Laplacien, C. R. Acad. Sci. Paris Ser. I 306 (1988), 171-175.

15. H. P. McKean and I. M. Singer, Curvature and eigenvalues of the Laplacian, J. Differential Geom. 1 (1967), 43-69.

16. J. Moser, A Harnack inequality for parabolic differential equations, Comm. Pure Appl. Math. 17 (1964), 101-134. Correction: 20 (1967), 231-236.

17. R. Seeley, $A$ sharp remainder estimate for the eigenvalues of the Laplacian in a domain in $\mathbf{R}^{3}$, Adv. in Math. 29 (1978), 244-269.

18. B. Simon, Nonclassical eigenvalue asymptotics, J. Funct. Anal. 53 (1983), 84-98.

19. H. Weyl, Das asymptotische Verteilunggesetz der Eigenwerte linear partieller Differentialgleichung, Math. Ann. 71 (1912), 441-479.

Department of Mathematics, University of Kentucky, LeXington, Kentucky 40506

E-mail address: rbrown@ms.uky.edu 